\title{
LA GEOMETRIZACIÓN DEL ESPACIO-MATERIA EN LA COSNIOLOGIA CARTESIANA
}

\author{
J. Cándido Martín \\ (Middlesex University, London)
}

La teoria de los vórtices de los movimientos planetarios ocupa un lugar fascinante en la historia de la ciencia. La primera edición de los Principia Philosophiae tiene lugar en 1644. La teoria de los vórtices surge para intentar dar respuesta a los cambios que se están produciendo en la Astronomía. Cuando Galileo apuntó su telescopio hacia la Luna contempló una naturaleza en los cielos idéntica a la de la tierra. Esto planteaba un nuevo problema a la astronomia: si los planetas y la tierra eran de idéntica naturaleza, los movimientos planetarios no podian seguir siendo explicados por medio del movimiento circular natural de los cuerpos perfectos, ahora se requeria una explicación en términos de física terrestre. Las esferas cristalinas serán sustituidas por un sistema de vórtices lluidos. El mérito de Descartes la consistido, como ha observado D'Alambert, en que el problema del mundo se ha reducido a problemas mecánicos (cfr. J.F. Scott, 1952, p. 181).

Al tiempo que Descartes finalizaba su primera obra sobre su concepción de la naturaleza, Le monde ou le traitè de la lumière, conocía la condena de Galileo y decidia no publicarla. Anos más tarde reelaborará un nuevo tratado en el que seguirá manteniendo la misma concepción de la naturaleza, los Principia Philosophine, que serian publicados en 1644. Descartes elaborará su propia propuesta cosmológica tratando de eliminar los principios artistotélicos, principios que ya habian sido, en parte, rechazados por Copémico, Galileo, Kepler, y otros. La nueva cosmología cartesiana ofrece un nuevo sistema del mundo en base a dos objetivos principales:

\begin{abstract}
El primero, materia y movimiento constituyen los componentes del musdo cartesiano. un mundo compuesto por tres elementos y que se regirá por tres leyes de la naturaleza que establecen la relación existente entre la materia y el movimiento. La explicación cartesiana será mecanicista. El segundo, en base a este mecanicismo, su máquina del mundo se extiende de manera ilimitoda, concibiendo 'mundos' en torno a cada estrella. El mundo cartesizno es plural. Frente a la unicidad de la cosmología aristotélica Descartes propondrá el concepto modemo de pluralidad de mundos, concepto ya anticipado en las filosofias de Cusa o de Bruno.
\end{abstract}

Descartes ha caracterizado a la materia eliminando de ella toda forma cualitativa, a partir de altora la naturaleza del cuerpo estará constituida por su extensión (longitud, anclura y profundidad), $y$ en modo alguno por su peso, dureza, color, o cualquier otra cualidad que afecte a los sentidos. Al eliminar las formas cualitativas la fisica cartesiana reduce la materia a componentes que pueden ser estudindos por la geometria. Descartes propone la explicación racional de los fenómenos de la naturaleza basado sobre la inteligencia humana y erigido sobre el modelo matemático frente a las 'qualitates occultac" empleadas libremente por los escolásticos (cfr. J.F. Scott, 1952, p. 158).

Esta concepción cartesiana del espacio-materia tiene dos consecuencias cosmológicas muy importantes. La primera de ellas consistiría en la imposibilidad del vacio. Carece de sentido, es más, sería contradictorio hablar de espacios vacios, puesto que la identilicación cartesiana entre espacio y materia no deja lugar al vacio. $Y$ de este planteamiento surgiria la segunda consecuencia, la concepción de un espacio ilimitado. Al igual que Nicolás de Cusa dos siglos antes (cfr. A. Koyré, Del mmdo cerrado, p. 115), Descantes reserva el concepto de inlinito para Dios, 
para aquello de lo cual tenemos una clara percepción de que es pertecto, el resto de las cosas carecen de esta perfección, y Descartes radicaliza la postura galileana expresada en Il Saggiatore de estudiar la naturaleza con el lenguaje de las matemáticas, un lenguaje que en el movimiento cartesiano deja a la luz lo esencial para el estudio geométrico: posición, dirección, trayectoria, relación funcional. Como dice A. Koyré en sus Estudios galileanos (1966, p. 317)

La geometrización a ultranza a la que sucumbe Descartes deshace la obra del tiempo -imagen ınovil de la eternidad innóvil-y yos presenta una inagen inmóvil y acabada del inacabamiento csencial del movimiento.

Movimiento que se torna relativo cundo trata de explicar el complejo movimiento terrestre, un movimiento más explicito en El mundo que en los Principia, y cuya complejidad radica en la reconciliación que bay que hacer de éste con la enseianza de la Iglesia (cfr. E.J. Aiton, 1972, p. 42).

El cosmos cartesiano se caracteriza por ser, en su mayor parte, liquido. Es un mundo compacto sin lugar para el vacio, pero en el que sus principales componentes están en constante movimiento, pues mícntras los cuerpos sólidos se caracterizan porque sus partes se tocan sin que queden espacios vacios entre ellas y ninguna se está moviendo, los liquidos están en constante movimiento, no permaneciendo sus partes unidas.

En la cosmologia cartesiana desaparecen las formas substanciales. La teoria de los vórtices ofrece una explicación general de los movimientos de los planetas en términos mecáni$\cos$. Además, en este universo ilimitado, donde se han destruido todos los principios clásicos, se destruye también la tesis de la unicidad del mundo. Frente al cosmos cerrado, uniforme e inmutable de la cosmologia aristotélica, Descartes lıa presentado un universo ilimitado y evolucionista, cada estrella es un mundo con planetas alrededor suyo, lo cual supone establecer el concepto actual de mundos. El océano cósmico cartesiano es la imagen más aproximada que podemos tener dal universo actual. El pluralismo es una consecuencia más de la nueva cosmologia propuesta por Descartes.

Descartes concibió la meta de la ciencia como el descubrimiento de una realidad objetiva que podia ser conocida con certeza. Tal realidad, creja Descartes, podia ser deducida por el método del razonamiento matemático a partir del principio axiomático de concevir ideas clara $y$ distintamente (cfr. E.J. Aiton, 1972, p. 34). El logro más signilicativo de la fisica cartesiana es la de enterrar a la herida fisica aristotélica; tras Descartes, la explicación de la realidad, de la naturaleza, se habrá deshecho de la fundamentación metafisica con la que el aristotelismo trataba de explicar la naturaleza.

La cosmologia cartesiana es sólo una consecuencia de sus principios físicos. El universo cartesiano es la antítesis del mundo clásico. Descartes es un punto de inflexión entre el enterrado mundo aristotélico y el emergente mundo moderno. El mayor logro de Descartes ha sido el de cerrar un libro y comenzar a escribir uno distinto. 


\section{BIBLIOGRAFí}

AITON, E.J. (1972), The vortex theory of planetary motions. London, Macdonald \& Co. BELAVAL, Y. (1960), Leibniz critique de Descartes. Paris, Gallimard.

BRUNSCIIVICG, L. (1937), Rene Descartes. Paris, Rieder.

DESCARTES, R., Oeures, Adam y Tannery $(A-T)$. Libreric Philosophique J. Vrin, Paris. 1974ss.

- (1991) El mundo o el tratado de la lus. Introd., trad. y notas por Ana Rioja. Madrid: Alianza Ed.,.

- (1995) Los principios de la Filosofia (P.F.). Introd., trad. y notas por Guillermo Quintás. Madrid: Alianza Ed.

KOYRÉ, A. (1957), Del mundo cerrado al universo infinito. Trad. de Carlos Solis Santos. Madrid: Siglo XXI, 1989 ( $7^{\supset}$ ed. esp.).

- (1966), Estudios Galileanos. Trad. de Mariano González Ambóu. Madrid: Siglo XXI, 1990 (5 ed. esp.).

SCOTT, J.F. (1952), The scientific work of Rene Descartas (1596-1650). London, Taylor \& Francis. 